\title{
Identificação de Jogos como Consequências de Comportamentos de Escolha para Crianças
}

\author{
Beatriz Coletti do Sacramento \\ Universidade Federal de São Carlos, São Carlos, SP, Brasil \\ Giovana Escobal ${ }^{1}$ \\ Celso Goyos \\ Instituto LAHMIEI, Departamento de Psicologia, Universidade Federal de São Carlos, \\ São Carlos, SP, Brasil
}

\begin{abstract}
Resumo
O comportamento social pode ser definido como comportamento de duas ou mais pessoas, uma em relação à outra, ou em conjunto em relação a um ambiente comum. O objetivo deste estudo foi identificar se fotos que representam jogos poderiam funcionar como reforçadores sociais para crianças típicas. Os participantes foram três crianças com desenvolvimento típico, sendo os estímulos experimentais duas figuras geométricas, e fotos de jogos (aplicativos) para Tablet. Foram realizadas avaliações de preferência diárias, em seguida à avaliação de preferência, após selecionar uma das figuras geométricas, era dada aos participantes a oportunidade de engajar-se no jogo com um dos experimentadores, representado na foto de sua maior preferência. A variável dependente foi a porcentagem de vezes que o participante escolheu um determinado formato geométrico por sessão. Os resultados sugerem que os procedimentos utilizados na presente pesquisa foram acurados para identificar jogos como reforçadores e seu valor reforçador foi testado posteriormente nas tarefas de discriminação simples e reversão. Esse procedimento pode ser conduzido de maneira rápida e com relativo baixo custo de resposta. A presente pesquisa demonstrou a importância em se oferecer oportunidades de escolha e oportunidades de contato com brincadeiras a crianças típicas, demonstrando que jogos podem ser usados como reforçadores em procedimentos de ensino.
\end{abstract}

Palavras-chave: Avaliação de preferência, aplicativos para tablet, valor reforçador, crianças.

\section{Identifying Games as Consequences of Choice Behavior for Children}

\begin{abstract}
Social behavior may be defined as the behavior of two or more people with respect to one another or in concert with respect to a common environment. The aim of this study was to identify if pictures that represent games could function as social reinforcers for children. Participants were three children with typical development, and the experimental stimuli were two geometrical figures and pictures that represented games (apps) for a Tablet. Daily preference assessments were made, following the preference assessment. After selecting a geometrical figure, the participants were given the opportunity to engage
\end{abstract}

1 Endereço para correspondência: Universidade Federal de São Carlos, Departamento de Psicologia, Instituto LAHMIEI, C.P. 676, São Carlos, SP, Brasil 13565-905. Fone: (16) 33518498, Fax (16) 33518361. E-mail: giovanaescobal@hotmail.com

Este manuscrito contou com apoio financeiro da Coordenação de Aperfeiçoamento de Pessoal de Nível Superior (CAPES). 
in the game with one of the experimenters, represented in their favorite picture. The dependent variable was the percentage of times the participant chose a particular geometrical figure per session. The results suggest that the procedures used in this research were accurate to identify games as reinforcers and its reinforcing value was subsequently tested in simple discrimination and reversal tasks. This procedure can be conducted quickly and with a relatively low cost of response. This research demonstrated the importance of offering choice opportunities and contact with games for typical children, showing that games can be used as reinforcers for teaching procedures.

Keywords: Preference assessments, tablet applications, reinforcing value, children.

\section{Identificación de Juegos como Consecuencias del comportamento de Selección para niños}

\section{Resumen}

El comportamiento social se puede definir como el comportamiento de dos o más personas con respecto a la otra, o conjuntamente, contra un entorno común. El objetivo de este estudio fue identificar si fotos que representan juegos podrían funcionar como refuerzos sociales para niños típicos. Los participantes fueron tres niños con un desarrollo típico, y los estímulos experimentales dos figuras geométricas, fotografías y juegos (aplicaciones) para la tableta. Evaluaciones diarias de preferencia se llevaron a cabo, siguiendo la evaluación de preferencia, después de seleccionar una de las figuras geométricas, se le dio a los participantes la oportunidad de participar en el juego con el experimentador 2, representada en la imagen de su mayor preferencia. La variable dependiente fue el porcentaje de veces que el participante eligió una forma geométrica determinada por sesión. Los resultados sugieren que los procedimientos utilizados en esta investigación fueron precisos para identificar los juegos como reforzadores y su valor de refuerzo se probó posteriormente en tareas de discriminación y de reversión de discriminación. Este procedimiento puede llevarse a cabo rápidamente y con costo de respuesta relativamente bajo. Esta investigación demostró la importancia de ofrecer oportunidades de elegir y oportunidades para jugar con los niños típicos, mostrando que los juegos pueden ser utilizados como refuerzos para los procedimientos de enseñanza.

Palabras clave: Evaluación de preferencia, applicaciones de tableta, valor reforzador, niños.

Para Keller e Schoenfeld (1950), o comportamento social pode ser descrito como aquele para o qual estímulos reforçadores ou discriminativos são, ou foram mediados pelo comportamento de outro organismo. Por 'mediado' diz-se 'em conexão com'. Para os autores, então, comportamentos sociais seriam as relações sujeito ambiente, nas quais os estímulos que constituem a relação comportamental são mediados por outros indivíduos (Andery \& Sério, 2006).

Para Skinner (1953/1965), "comportamento social pode ser definido como comportamento de duas ou mais pessoas, uma em relação à outra, ou em conjunto em relação a um ambiente comum" (p. 297). Está implícito nessa definição que, no caso, o comportamento de um indivíduo é mediado por respostas de um outro indivíduo, ou seja, que respostas deste segundo individuo assumem funções de estimulo - como já afirmaram Keller e Schoenfeld (1950). Para tanto, as respostas dos dois participantes podem estar envolvidas em uma única contingência, possivelmente delimitada pelos estímulos discriminativos que evocam as respostas de cada um deles em conjunto, e pelos estímulos reforçadores, que só são produzidos se ambos emitirem as respostas que fazem parte da contingência. Nesta contingência, além disso, o comportamento de cada um dos participantes, opera como estímulo discriminativo ou reforçador para o comportamento do outro (Andery \& Sério, 2006). 
Ademais, Sampaio e Andery (2010) ressaltam tal definição de Skinner (1953/1965, 1957/1992) de restringir o comportamento social a contingências tríplices cujas consequências são produzidas pelo comportamento operante do outro indivíduo, ou seja, as consequências são produzidas por uma ação de um indivíduo, porém, há a mediação de outro indivíduo. Segundo os autores ainda, o comportamento social seria "qualquer contingência tríplice cujas consequências são mediadas pelo comportamento do outro". Logo, a produção de consequências envolve não só o comportamento operante do indivíduo em questão, mas também o comportamento operante de um segundo indivíduo que está mediando tal consequência.

Além da compreensão do termo utilizado "comportamento social", faz-se importante falar sobre os três tipos de pesquisas desenvolvidos pela Análise do Comportamento: Básica, Translacional e Aplicada. A primeira também conhecida como Análise Experimental do Comportamento (Experimental Analysis of Bahavior - EAB) foi descrita por Poling (2010) como fraca no esforço coletivo para um melhor impacto social, acrescentando ainda, que estudos da pesquisa básica não seriam relevantes para ações significativas de cada indivíduo em seus ambientes naturais. Além disso, a EAB aparece mais frequentemente focada na teoria e em princípios fundamentais do que em problemas sociais (Critchfield, 2011).

Já a pesquisa Translacional preocupa-se com problemas práticos, porém, trabalha no âmbito de convencer a sociedade que a pesquisa básica merece seu apoio. Ademais, tal pesquisa se preocupa em explorar a relevância social de determinado estudo, sem comprometer a capacidade do investigador em explorar problemas teóricos. Portanto, um dos objetivos da pesquisa Translacional é integrar as descobertas da pesquisa básica com suas possíveis implicações (Critchfield, 2011).

De maneira prática, a pesquisa Aplicada para Baer, Wolf, e Risley (1968) deixa evidenciada a importância da mudança comportamental, bem como suas características quantitativas, suas manipulações experimentais as quais anali- sam de maneira clara o que foi responsável pela mudança comportamental, a descrição de todos os procedimentos que contribuíram para a mudança, e a efetividade desses procedimentos, e por último, a capacidade de generalização dessa mudança. Como a pesquisa aplicada não prescinde do rigor científico e do controle de variáveis, o que a diferencia da pesquisa básica não são esses aspectos, mas sim a relevância social da relação entre as variáveis investigadas.

De modo geral, a pesquisa Translacional é concebida como a exploração de fatores que pesquisadores aplicados podem derivar da ciência básica, e de maneira mais clara, Poling (2010) ressalta o ponto sobre os pesquisadores básicos depositarem a responsabilidade da pesquisa translacional em selecionar problemas de pesquisa que estão diretamente ligados à importantes problemas sociais. Outro fator relevante aos tipos de pesquisas e suas correlações, se deve ao fato de que pesquisadores básicos devem entender os problemas práticos bem o bastante para então, decidirem quais aspectos da pesquisa básica, bem como quais princípios básicos serão relevantes a eles. A colaboração com pesquisadores aplicados é uma boa maneira para os pesquisadores básicos em melhorar seu pensamento translacional (Mace \& Critchfield, 2010).

Ao mesclarmos as ideias previamente expostas de comportamentos sociais e tipos de pesquisas na Análise do Comportamento expomos a importância em se pensar procedimentos relevantes para tais comportamentos. Por exemplo, para crianças que apresentariam algum déficit em comportamentos sociais, pode-se pensar em uma intervenção focada no indivíduo através de técnicas de aprendizagem social e comportamentais (Cooper, Griffith, \& Filer, 1999), o engajamento em comportamentos sociais tem sido relatado como um componente efetivo desses tipos de intervenções para muitas disfunções infantis incluindo a fobia social infantil (Spence, Donovan, \& Brechman-Toussaint, 1999) e habilidades de aprendizagem específica (Forness \& Kavale, 1999).

Para a realização de tais intervenções, a identificação de estímulos reforçadores é um aspecto importante para a efetividade de programas deli- 
neados para facilitar a aquisição de habilidades. Adicionalmente, o sucesso de programas com reforçamento positivo delineados para diminuir a ocorrência de comportamentos inadequados, poderá vir a ser efetivo somente se tais reforçadores utilizados possuírem valor reforçador. $\mathrm{E}$ a identificação de tais reforçadores considerados potentes é um desafio. A maioria dos estudos em identificação de reforçadores tem focado em: identificação de reforçadores em potencial (e.g. avaliações de preferência) sem testar se o estímulo pode ser usado para aumentar a resposta; avaliar os efeitos reforçadores do estímulo (e.g. avaliação de reforçadores) sem um procedimento para dizer qual estímulo específico funcionaria como reforçador (Piazza, Fisher, Bowman, \& Blakeley-Smith, 1999).

Apesar dos imensos benefícios de se oferecer oportunidade de escolha, essas dificilmente são incluídas nos procedimentos de ensino (Reid, Parsons, Green, \& Browning, 2001). Para tanto, um procedimento útil para tais comportamentos é a avaliação de preferência, pois quanto maior o conhecimento sobre as preferências de uma pessoa, melhores são as condições de motivá-la com sucesso a realizar uma tarefa (Escobal, Macedo, Duque, Gamba, \& Goyos, 2010). Porém, ao mencionarmos avaliação de preferência como procedimento avaliativo de comportamentos sociais, é preciso utilizar-se de um formato alternativo para a realização de tal procedimento. Por exemplo, a representação de certas brincadeiras ou jogos em figuras para a realização da avaliação de preferência. Essas brincadeiras ou jogos sendo identificadas poderiam então, serem pareados com episódios sociais naturais para reforçar a aquisição de comportamentos sociais. A seguir seguem alguns estudos que se pautaram em métodos tradicionais para avaliarem a preferência dos sujeitos experimentais.

Conyers et al. (2002) conduziram um estudo para avaliar como as respostas de escolha eram influenciadas por modalidades diferentes de estímulos (objetos, figuras e descrições verbais). Foram apresentadas oportunidades de escolha para nove indivíduos com deficiência intelectual em uma avaliação de preferência com estímulos pareados. Cada par de itens era apresentado para cada participante em três condições (itens tangíveis, figuras desses itens e os nomes) usando um delineamento de reversão. Primeiramente, a avaliação foi conduzida com itens comestíveis, e depois com itens não comestíveis. Os participantes também foram expostos a um teste para medir suas habilidades em tarefas de discriminação simples e condicional. Eles selecionaram seus itens preferidos consistentemente tanto com figuras quanto com objetos e suas habilidades de fazer essas escolhas foram previstas com $94 \%$ de acurácia pelas habilidades de discriminação. Os achados sugerem que a modalidade dos estímulos pode afetar a acurácia de uma avaliação de preferência, e que a revisão sistemática de habilidades básicas de discriminação pode ser usada para predizer a efetividade de métodos diferentes para essa população.

Groskreutz e Graff (2009) apresentaram diferentes resultados, nos quais os mesmos itens foram avaliados em três formatos diferentes de avaliação de preferência: avaliação tangível com acesso ao item, avaliação com figuras com acesso ao item, e avaliação com figuras sem acesso ao item. Algo importante foi feito nesse estudo, os experimentadores testaram o emparelhamento entre cada figura e seu item tangível correspondente, podendo esta ser uma habilidade necessária quando se utilizar qualquer procedimento similar (figuras no lugar de itens tangíveis). Foi apontado também, que na primeira sessão de avaliação de preferência com figuras sem acesso ao item os participantes emitiram comportamentos inadequados. E um dos resultados relevantes que os autores encontraram foi que, quando os resultados entre os diferentes formatos de avaliação de preferência são diferentes, as avaliações que incluem acesso ao item escolhido é o melhor indicador das reais propriedades reforçadoras do estímulo.

É importante salientar a importância de mais estudos que determinem como se estabelecer a oportunidade de escolha como reforçadora para indivíduos cujo comportamento parece não ser afetados por elas. Por exemplo, a escolha deve adquirir propriedades reforçadoras depois que os indivíduos forem expostos repetidamente a situações de escolha e não-escolha da seguinte 
maneira: quando a escolha está disponível, o indivíduo pode obter itens altamente preferidos; quando a escolha estiver ausente, o indivíduo poderá obter somente itens não preferidos. Se a escolha puder ser estabelecida como um reforçador, professores e clínicos deverão apresentar uma estratégia de tratamento adicional para indivíduos com deficiências severas e profundas. Adicionalmente, tais descobertas ressaltariam a importância de prover frequentes oportunidades de escolha aos indivíduos (Lerman et al.,1997).

Nuernberger, Smith, Czpar, e Klatt (2012) investigaram a preferência por brincadeiras ('cavalinho', 'vai cair', 'pega-pega', 'gira-gira', 'porquinho', 'não pode levantar', 'cócegas', 'madeira', 'foguete', 'preparar, apontar, fogo', 'balanço' e 'carrossel') em três crianças com autismo. Foram realizadas avaliações de preferência (num formato alternativo) com múltiplos estímulos sem reposição para determinar uma hierarquia de preferência para as brincadeiras, seguida de uma avaliação de reforçador. Foi solicitado aos pais e terapeutas que identificassem quais brincadeiras eram mais preferidas para cada criança. Feito isso, eram apresentadas fotos do experimentador realizando determinadas brincadeiras com a criança, e era solicitado que ela escolhesse uma. Após identificadas as brincadeiras mais preferidas por cada participante, foi realizada a avaliação de reforçador em que era solicitado aos participantes que realizassem tarefas simples como, por exemplo, colocar dois garfos e dois canudos em recipientes apropriados. Respostas corretas eram reforçadas pelas brincadeiras determinadas na avaliação de preferência feita anteriormente. Os resultados mostraram que diferentes tipos de brincadeiras funcionaram como reforçadores. No estudo apenas duas avaliações de reforçadores foram realizadas para cada brincadeira, o que pode ter prejudicado seus resultados. Mais sessões de avaliações de reforçadores poderiam permitir avaliar o responder mais acuradamente para cada brincadeira preferida. Adicionalmente, apenas um formato de avaliação de preferência foi testado neste estudo.

$\mathrm{O}$ uso de recursos de computador pode fornecer um uso mais frequente de avaliações de preferência por um número maior de pessoas, além de apresentação de registro mais acurado e economia de tempo. Snyder, Higbee, e Dayton (2012) relatam que vídeos, por exemplo, podem ser um formato eficaz para a apresentação de estímulos sociais ou complexos nas avaliações de preferência. Em seu estudo, avaliaram a correspondência entre hierarquias de preferências geradas a partir de avaliações de preferência com brinquedos ou vídeos dos brinquedos. Itens altamente reforçadores corresponderam nas duas avaliações para cinco dos seis participantes, e itens classificados como de baixa preferência corresponderam para quatro participantes. O estudo teve como foco avaliar a validade das avaliações de vídeo, comparando os resultados com os de avaliações tangíveis, e avaliar a preferência para brinquedos em vez de estímulos complexos. Os autores sugeriram que para avaliar a utilidade clínica de vídeos, pesquisas futuras poderiam investigar seu uso com estímulos sociais ou complexos. Apontam também que uma avaliação da consequência não foi realizada em seu estudo para confirmar se os estímulos identificados como de alta preferência funcionavam como reforçadores.

A presente pesquisa, além de identificar se jogos computadorizados representados por fotos funcionavam como reforçadores, testou a efetividade de tais jogos em tarefas de discriminação simples e reversão da discriminação, e realizou uma fase de emparelhamento foto-jogo, considerada uma variável importante e que influencia os resultados quando não realizada. $\mathrm{O}$ objetivo desse estudo, assim, foi avaliar os efeitos reforçadores de jogos (aplicativos para Tablet) de alta preferência, representados por fotos, sobre a aquisição de discriminações simples e reversão da discriminação por crianças com desenvolvimento típico.

\section{Método}

\section{Participantes}

Participaram desse estudo três crianças com desenvolvimento típico, com idade de quatro anos, sendo uma menina (Mariza) e dois meninos (André e Milton), recrutados em uma escola 
municipal no interior do estado de São Paulo. Os nomes utilizados para os participantes são fictícios seguindo as orientações do comitê de ética pelo qual foi submetida a pesquisa previamente ao seu desenvolvimento. Um pré-requisito para a participação foi de que os indivíduos apresentassem entendimento de instruções simples (e.g., mantivessem contato visual, olhassem quando fossem chamados, emitissem comportamento de escolha, etc.) e não tivessem familiaridade com procedimentos de avaliação de preferência. A coleta de dados foi realizada durante o período letivo dos participantes.

\section{Procedimento Éticos}

Os procedimentos éticos aprovados pelo Comitê de Ética da Universidade Federal de São Carlos, Processo n ${ }^{\circ}$ 21737813.6.0000.5504, Parecer no 469.136, termo de consentimento esclarecido e autorização (assinados pelos responsáveis pelas crianças), foram seguidos.

Tabela 1

Jogos do Experimento e suas Figuras Correspondentes

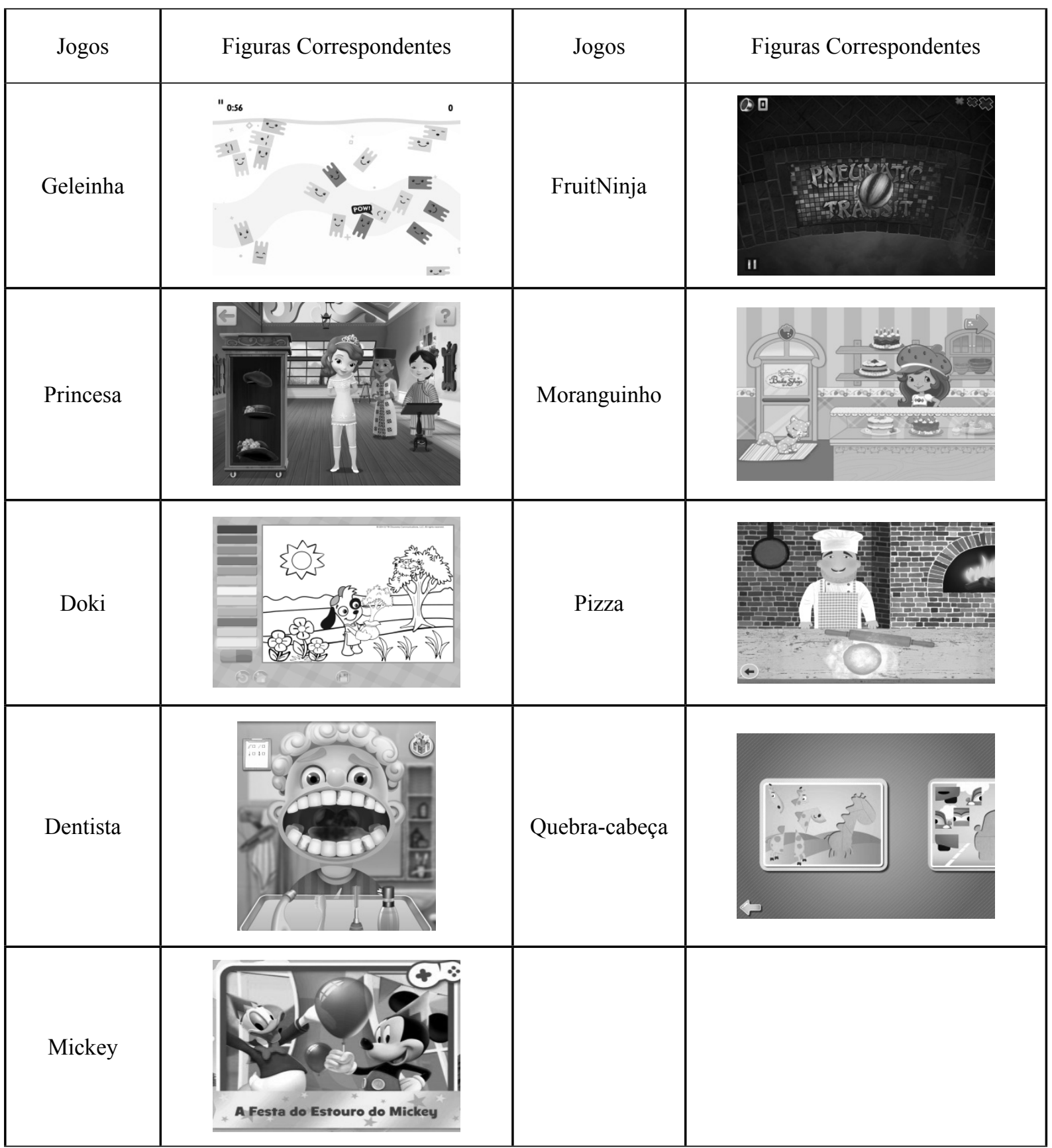




\section{Local e Recursos Materiais}

A pesquisa foi realizada na escola em que os participantes foram recrutados, em uma sala de aproximadamente $3 \mathrm{~m} \times 4 \mathrm{~m}$, durante o período letivo. Foram utilizados um computador portátil "touch screen" com software MestreLibras (Elias \& Goyos, 2010), um Tablet, uma mesa, uma cadeira, papel, caneta, folhas de registro, papel fotográfico HP Premium Plus, cronômetro, máquina fotográfica e filmadora. O software MestreLibras foi utilizado para programar e gerenciar as sessões, registrar e arquivar os dados.

\section{Estímulos Experimentais}

Foram utilizadas nove figuras correspondentes de nove jogos (aplicativos) para Tablet. As figuras apresentadas eram coloridas, com as dimensões de $11 \mathrm{~cm}$ de largura e $9 \mathrm{~cm}$ de altura
(Tabela 1). [Estes estímulos foram representados pela sigla $\mathrm{S}+$ ao longo da pesquisa e utilizadas nas etapas de Familiarização (Fase II), Tarefa de Matching (Fase III) e Avaliação de Preferência (Fase IV). Em sua presença, a emissão de uma resposta era reforçada].

Adicionalmente, foram utilizados dois estímulos experimentais representados por figuras geométricas de círculo e quadrado, em traçado preto sobre o fundo branco, no formato Joint Photographic Experts Group (JPEG; Tabela 2). [Estes estímulos foram representados pela sigla $\mathrm{S}+$ e S- ao longo da pesquisa e utilizados nas fases de discriminação simples e reversão da discriminação simples -Teste do efeito reforçador dos itens de preferência (Fase V). Na presença do $\mathrm{S}+$ a emissão de uma resposta era consequenciada com jogos; na presença do S- a emissão de uma resposta não era consequenciada por jogos].

Tabela 2

Distribuição dos Estímulos Experimentais utilizados na Tarefa de Discriminação Simples Envolvendo Formatos Geométricos

\begin{tabular}{|c|c|c|}
\hline Tentativa & Posição Esquerda & Direita \\
\hline 1 & $\mathrm{~S}+$ & S \\
\hline 2 & $\mathrm{~S}+$ & s \\
\hline 3 & S- & S \\
\hline 4 & $\mathrm{~S}+$ & S \\
\hline 5 & S- & $\mathrm{S}$ \\
\hline 6 & S- & , \\
\hline 7 & $\mathrm{~S}+$ & S \\
\hline 8 & & \\
\hline
\end{tabular}




\section{Procedimento}

As etapas ocorreram na seguinte ordem:

Tabela 3

Estratégia Experimental da Pesquisa

\begin{tabular}{ccl}
\hline $\begin{array}{c}\text { Fase } \\
\text { Experimental }\end{array}$ & Fases & \multicolumn{1}{c}{ Função } \\
\hline I & Entrevista & $\begin{array}{l}\text { Entrevistar pais e professora para levantar quais aplicativos do } \\
\text { Tablet eram de interesse para cada participante. } \\
\text { Apresentação individual de cada figura que representava um } \\
\text { aplicativo para que o sujeito entrasse em contato com os estímulos } \\
\text { experimentais. }\end{array}$ \\
II & Familiarização & $\begin{array}{l}\text { Prover um maior controle com relação ao pareamento figura-jogo } \\
\text { por parte do participante. O critério para passar para a próxima } \\
\text { etapa foi de 100\% de acertos. } \\
\text { Avaliar qual jogo era de maior preferência pelo participante, para } \\
\text { que o mesmo pudesse ser utilizado na fase seguinte. }\end{array}$ \\
IV & Tarefa de Matching & $\begin{array}{l}\text { Teste do efeito reforçador } \\
\text { Essa etapa consistiu em avaliar o valor reforçador do jogo escolhido } \\
\text { como de nível alto de preferência na fase de Multiple Stimulus } \\
\text { Without Replacement (MSWO). }\end{array}$ \\
\hline
\end{tabular}

Entrevista. Primeiramente, foi realizada uma entrevista com os pais e a professora dos participantes, para levantar quais aplicativos do Tablet eram de interesse para cada participante dentre nove opções apresentadas. Quatro foram selecionadas para cada participante para a realização da fase de familiarização.

Familiarização. Essa etapa consistiu em apresentação individual de cada uma das quatro figuras que representavam um aplicativo pelo Experimentador 1, sendo dito concomitantemente à apresentação o nome do aplicativo representado na figura apresentada, seguida da atividade por 15 segundos (brincar com o aplicativo apresentado). O brincar com o jogo era sempre realizado com o Experimentador 2, uma vez este jogava, e outra vez o participante jogava. Foram realizadas duas sessões dessa etapa durante dois dias consecutivos, somando um total de quatro sessões de familiarização. Essa etapa foi importante para que o sujeito entrasse em contato com os estímulos experimentais.

Tarefa de Matching. Essa etapa foi realizada para que houvesse um maior controle com relação ao pareamento figura-jogo por parte do participante. Ela consistiu em: o participante brincava com um dos jogos já utilizados na fase anterior (Familiarização), juntamente com o Experimentador 2, e após 15 segundos de brincadeira, as quatro figuras (escolhidas pelos pais e professores na fase de entrevista) que representavam os jogos do Tablet eram colocadas sobre a mesa pelo Experimentador 1, seguidas da pergunta "Com qual você brincou?", e a criança apontava para uma das figuras. Isso foi feito com os quatro jogos, sendo realizada em duas sessões por dia, com quatro tentativas cada, em dois dias diferentes, totalizando quatro sessões para essa etapa. O critério para passar para a próxima etapa foi de $100 \%$ de acertos.

Avaliação de Preferência. Foi realizada uma Avaliação de Preferência com Múltiplos Estímulos sem reposição (MSWO - Multiple Stimulus Without Replacement; Carr, Nicolson, \& Higbee, 2000; De-Leon \& Iwata, 1996), na qual as quatro figuras que representavam os jogos (aplicativos) no Tablet eram disponibilizadas sobre a mesa. O Experimentador 1 dizia "Escolha", 
o participante apontava para uma das figuras, e imediatamente recebia do Experimentador 2 o jogo que escolheu por 15 segundos (ou seja, o participante brincava com o experimentador por 15 segundos com aquele jogo). O Experimentador 1 então, registrava a resposta, retirava a figura escolhida pelo participante, e rearranjava as três figuras restantes sobre a mesa, fornecendo novamente a instrução "Escolha". Novamente o jogo escolhido em forma de figura era fornecido pelo Experimentador 2 por 15 segundos. Isso foi realizado até que o participante escolhesse cada uma das quatro figuras. $\mathrm{O}$ jogo tido como de nível alto de preferência foi sempre o jogo representado pela figura que o participante escolheu primeiro. Esse jogo foi utilizado na etapa seguinte. Foi realizada apenas uma sessão de avaliação de preferência no primeiro dia após as etapas anteriores, previamente à primeira sessão da Tarefa de discriminação.

Teste do Efeito Reforçador dos Itens de Preferência. Essa etapa consistiu em avaliar o valor reforçador do jogo escolhido como de nível alto de preferência na fase de MSWO. Essa etapa consistia em Fase 1 e Fase 2. Cada sessão consistiu em um bloco com quatro tentativas. Cada tentativa era iniciada pela apresentação concomitante de dois estímulos, o círculo e o quadrado, na tela do computador, ocupando cada um dos estímulos os cantos inferiores, esquerdo e direito da tela. As posições em que os estímulos eram apresentados variavam de maneira randomizada ao longo das tentativas. Em seguida à apresentação dos estímulos a instrução oral "Escolha" era apresentada. Na Fase 1, respostas ao $\mathrm{S}+$ (círculo), definido como o estímulo de mais alta preferência no teste de avaliação de preferência, foram seguidas por 15 segundos de acesso da brincadeira representada na foto escolhida como de nível alto de preferência no teste de avaliação de preferência realizado anteriormente, e respostas ao S- (quadrado) foram seguidas por 15 segundos de 'tela preta' e pela tentativa seguinte.

Após atingir estabilidade nos resultados na Fase 1, foi realizada a Fase 2, com o procedimento semelhante à fase anterior, porém houve a reversão dos estímulos, o $\mathrm{S}+$ passou a ser re- presentado pela escolha no quadrado, e o S-, pela escolha no círculo. As brincadeiras fornecidas após as escolhas no $\mathrm{S}+$, tanto na Fase 1, quanto na Fase 2, eram fornecidas pelo Experimentador 2.

Foram realizadas de duas a três sessões por dia, em uma média de seis dias de trabalho.

\section{Delineamento Experimental}

Foi utilizado um delineamento experimental de sujeito único (Smith, 2012), para verificar se as brincadeiras e jogos identificados como de maior preferência alteraram a porcentagem de escolhas na tarefa de escolha envolvendo formatos geométricos diferentes. Foram feitas comparações intra e intersujeitos (Tawney \& Gast, 1984).

\section{Procedimento para Registro e Análise de Dados e para Cálculo de Fidedignidade}

Os dados de interesse foram as respostas de escolha nos esquemas concorrentes. Para a análise do comportamento de escolha, a variável dependente foi a porcentagem de escolha para cada estímulo. A porcentagem foi calculada dividindo-se a soma do número de escolhas para cada figura por avaliação pelo total de oportunidades de escolhas nas duas avaliações. Adicionalmente, foi avaliado o número de respostas corretas nas tarefas de escolha envolvendo formatos geométricos diferentes, bem como se o jogo teve função reforçadora, influenciando o desempenho (medido por meio do parâmetro número de acertos) na tarefa. Os dados foram coletados a partir dos registros, de $100 \%$ das tentativas, realizadas pela ferramenta informatizada e por meio de protocolos para registro observacional das escolhas - Folha de Registro. Tais observações e registros, referentes ao cálculo de fidedignidade foram feitos pelo experimentador e por um observador independente (ou Experimentador 3), treinado para fins de teste de confiabilidade. $\mathrm{O}$ cálculo de fidedignidade foi obtido através da fórmula: número de concordância entre os dois observadores, dividido pelo número de concordância mais discordância, multiplicado por 100 (Hall, 1974), e o resultado foi de $91,9 \%$. 


\section{Resultados}

Seguem os resultados de cada etapa do referido Experimento.

\section{Familiarização e Tarefa de Matching}

Foram realizadas quatro sessões de Familiarização, duas sessões por dia. Portanto, todos os jogos da lista de cada participante foram apresentados aos mesmos, em um total de quatro vezes. O critério para fase de Tarefa de Matching foi de $100 \%$ de acerto dos participantes no pareamento figura-jogo no Tablet. Foram realizadas quatro sessões, duas sessões por dia. Todos os participantes atingiram critério nessa fase.

\section{Avaliação de Preferência}

Foi realizada apenas uma avaliação de preferência com múltiplos estímulos sem reposição (MSWO) para cada participante, logo após o término da etapa de Tarefa de Matching. Para André, o jogo 'FruitNinja' foi dado como de alto nível de preferência; para Mariza e para Milton, o jogo considerado como de alto nível de preferência foi 'Quebra-cabeça'.

\section{Avaliação do Efeito Reforçador dos Itens de Preferência}

Essa etapa foi realizada para avaliar a função reforçadora dos estímulos. Dois dos três participantes, André e Mariza, obtiveram os resultados esperados de acordo com o objetivo do presente estudo, pois os estímulos utilizados funcionaram como reforçadores em tarefas de discriminação simples (Figura 1).

Na Fase 1, André recebia o jogo 'FruitNinja' por 15 segundos, após a escolha do S+, e recebia 15 segundos de 'tela preta', seguidos da próxima tentativa quando escolhia o S-. Em cinco sessões com quatro tentativas cada, André apresentou desempenho estável em 100\% de escolhas no $\mathrm{S}+$. Na Fase 2, introduziu-se a reversão da discriminação, tendo sido apresentada a estabilidade nos resultados em $100 \%$ após duas sessões.

Para Mariza, na Fase 1, foram realizadas oito sessões para alcançar a estabilidade em $100 \%$. A participante recebia o jogo 'Quebra-cabeça' após a escolha no $\mathrm{S}+$, e 15 segundos de 'tela preta', mais a apresentação da próxima tentativa, após a escolha no S-. Mariza apresentou $50 \%$ de escolhas tanto no $\mathrm{S}+$, quanto no $\mathrm{S}-$, nas duas primeiras sessões, apresentando $75 \%$ de escolhas no $\mathrm{S}+$ nas próximas três próximas sessões. Nas próximas sessões atingiu estabilidade no desempenho apresentando $100 \%$ de escolhas no S+. Na Fase 2, com a reversão da discriminação, a estabilidade ocorreu mais rapidamente apresentando três sessões de $75 \%$ de escolhas no S+, e $25 \%$ no S-, e, então, apresentou estabilidade no desempenho apresentando $100 \%$ de escolhas no $\mathrm{S}+$ nas quatro últimas sessões.

Já para Milton, não foi possível a realização da Fase 2, pois esse não apresentou estabilidade dos dados durante a Fase 1 do experimento. $\mathrm{O}$ jogo utilizado como consequência para as escolhas no S+ foi 'Quebra-cabeça'. Portanto, ao escolher o $\mathrm{S}+$, ele recebia 15 segundos deste jogo, e ao escolher o S-, recebia 15 segundos de 'tela preta' seguidos da apresentação da próxima tentativa. Este participante apresentou instabilidade em seus dados, nas três primeiras sessões, mantendo-se em 50\% de escolhas tanto no $\mathrm{S}+$, quanto no S-. A partir da Sessão 14, houve o aumento do número de tentativas por sessões para esse participante, ao invés de quatro tentativas, eram realizadas 12 tentativas por sessão.

$\mathrm{Na}$ Sessão 10, o participante apresentou $100 \%$ de escolhas no S+, o que não se manteve, apresentando desempenho instável da Sessão 12 à Sessão 18, apresentando uma queda nas escolhas para o $\mathrm{S}+$ nas últimas sessões. Levantando-se a hipótese de que se tivessem mais sessões, provavelmente, as escolhas no $\mathrm{S}+$ estabilizariam em $0 \%$ de escolhas. Essa hipótese não pode ser comprovada, pois houve o encerramento do ano letivo. 

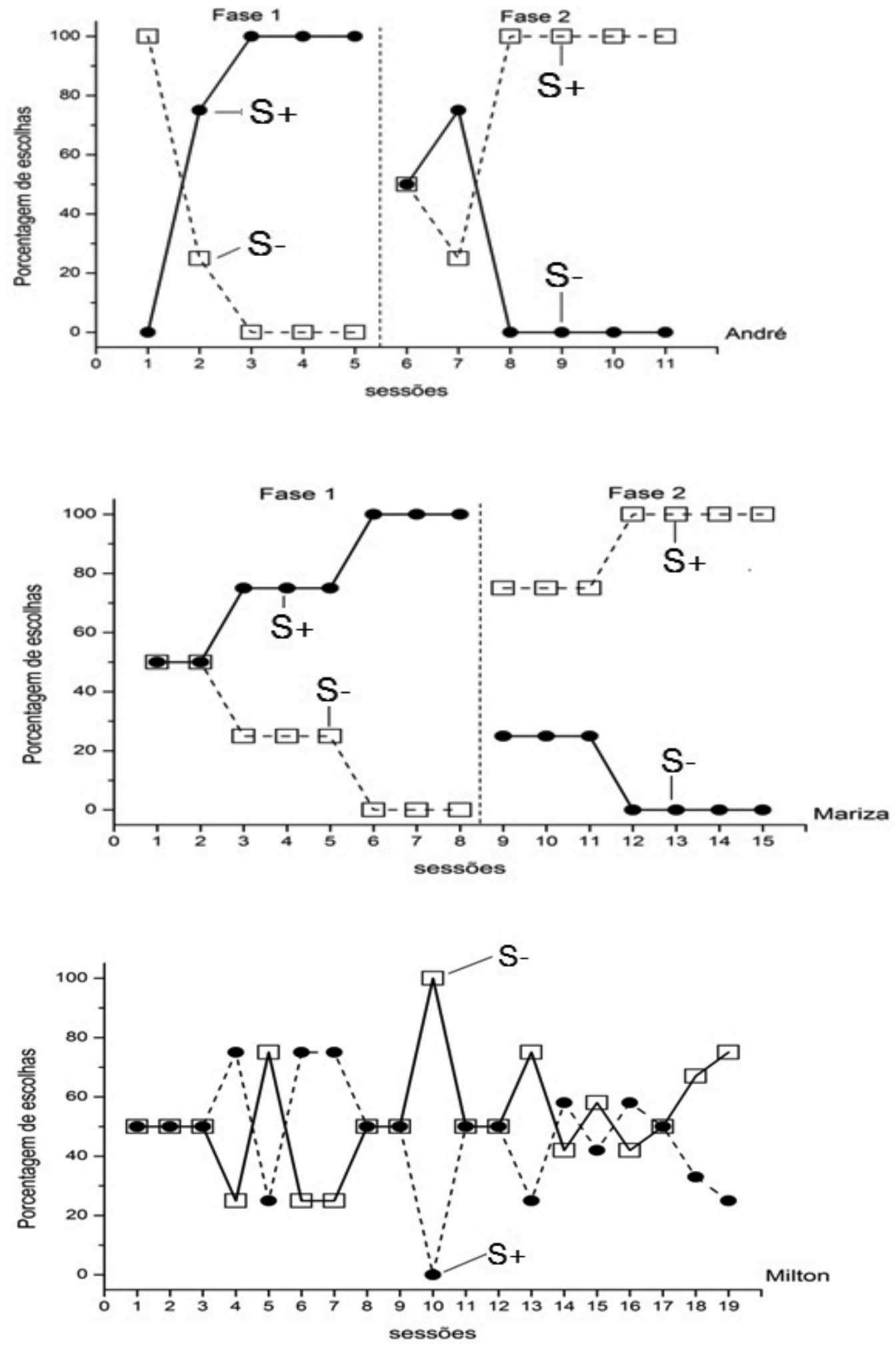

Figura 1. Porcentagem de escolhas dos participantes André, Mariza e Milton na tarefa de discriminação simples envolvendo formatos geométricos diferentes. Figuras abertas representam escolhas no S- e figuras preenchidas representam escolhas no $\mathrm{S}+$ na Fase 1. Figuras abertas representam escolhas no $\mathrm{S}+\mathbf{e}$ figuras preenchidas representam escolhas no S- na Fase 2. 


\section{Discussão}

Pautado na descrição feita na Introdução dos três tipos de pesquisa utilizados em Análise do Comportamento, pode-se explorar que no presente estudo tem-se o rigor experimental proveniente da pesquisa básica, com um setting similar ao do laboratório, e com a preocupação de realização em condições mais naturais de um relevante comportamento. Além disso, a pesquisa preocupou-se em manter um rigor experimental, analítico, tecnológico, bem como apresentar resultados efetivos e com possibilidade de generalização.

Ao se pensar na consequência social como reforçador condicionado, as consequências sociais poderiam inicialmente ser pareadas com reforçadores primários até elas adquirirem valor reforçador condicionado e depois seriam retirados os reforçadores primários, esse procedimento poderia ser realizado em estudos futuros (Lerman et al., 1997). A utilização do procedimento de Matching, muito provavelmente, fez com que as fotos dos jogos adquirissem valor reforçador condicionado, além de atestar mais confiabilidade ao estudo, demonstrando a possibilidade de mais controle em pesquisas translacionais e aplicadas. Isto é de fundamental importância em intervenções e pesquisas aplicadas (Barnes, 1994; Barnes-Holmes, \& Barnes-Holmes, 2000).

No estudo de Conyers et al. (2002), foi sugerido que a modalidade dos estímulos pode afetar a acurácia de uma avaliação de preferência, e que a revisão sistemática de habilidades básicas de discriminação pode ser usada para predizer a efetividade de métodos diferentes para essa população. No presente estudo, a modalidade de estímulos utilizada parece ter sido adequada em função da estabilidade dos dados de dois dos três participantes na fase de Avaliação do efeito reforçador dos itens de preferência.

Groskreutz e Graff (2009) apresentaram como resultados relevantes que quando os resultados entre os diferentes formatos de avaliação de preferência são diferentes, as avaliações que incluem acesso ao item escolhido é o melhor indicador das reais propriedades reforçadoras do estímulo. Groskreutz e Graff (2009) também pré-testaram habilidades de emparelhamento entre cada figura e o seu item tangível correspondente, o que pode ser uma habilidade necessária ao substituir itens tangíveis por figuras. No presente estudo, realizou-se um procedimento para que houvesse o emparelhamento dos jogos com suas respectivas figuras, o que se mostrou um controle experimental importante, pois consistências nas escolhas demonstraram que o jogo e a figura podem ser tomados um pelo outro.

Adicionalmente, ao escolher a figura na avaliação de preferência, o participante recebia do experimentador o jogo propriamente dito, e não a sua figura correspondente, o que se mostrou importante indicando as reais propriedades reforçadoras do estímulo. Higbee, Carr, e Harrison (1999) realizaram uma pesquisa para determinar a possibilidade de usar figuras em avaliações de preferência. Na avaliação com itens tangíveis, cada vez que o participante selecionava um item, ele ganhava $20 \mathrm{~s}$ de acesso a esse item. $\mathrm{Na}$ avaliação com os cartões, entretanto, a resposta de seleção não resultava no acesso ao item correspondente. Segundo os autores, é importante fornecer o acesso ao item, pois se o acesso ao estímulo for concebido como o reforçador que mantem a resposta de seleção, então, não fornecer acesso ao item seria similar a operação de extinção.

Para Vollmer e Hackenberg (2001), as consequências sociais podem estar associadas com aspectos incondicionados. Diferente de itens tangíveis que podem ser entregues de forma padronizada, as consequências sociais podem vir em uma variedade grande de formatos - expressões faciais, contatos sociais, vocalizações sendo liberadas de maneiras diferentes. O maior desafio está em descobrir as características que contribuem para a efetividade das consequências sociais. Van Houten, Nau, Mackenzie-Keating, Sameoto, e Colavecchia (1982), encontraram que reprimendas verbais eram mais efetivas em suprimir os problemas quando eram combinadas com contato físico e visual do que somente as reprimendas verbais sozinhas. Kazdin e Klock (1973) encontraram que sorrisos e contato físico aumentaram a efetividade reforçadora das aprovações verbais na modificação do comportamen- 
to de alguns alunos em sala de aula. Portanto, ao se trabalhar com determinados comportamentos sociais deve-se elaborar minuciosamente o procedimento ao ser utilizado, e a relevância de uma tarefa de Matching ao se trabalhar com tais consequências. Dessa forma, pode-se pautar os resultados obtidos em uma aplicação prática, a partir de fundamentos básicos da Análise do Comportamento.

Mais estudos deveriam determinar como se estabelecer a oportunidade de escolha como reforçadora para indivíduos cujo comportamento parece não ser afetados por elas. Por exemplo, a escolha deve adquirir propriedades reforçadoras depois que os indivíduos forem expostos repetidamente a situações de escolha e não-escolha da seguinte maneira: quando a escolha está disponível, o indivíduo pode obter itens altamente preferidos; quando a escolha estiver ausente, o indivíduo poderá obter somente itens não preferidos. Se a escolha puder ser estabelecida como um reforçador condicionado, professores e clínicos deverão apresentar uma estratégia de tratamento adicional para indivíduos com deficiências severas e profundas. Adicionalmente, tais descobertas ressaltariam a importância de prover frequentes oportunidades de escolha aos indivíduos (Lerman et al.,1997).

Com relação aos dados de indiferença de um dos participantes, pode-se discorrer sobre as (a) possíveis contingências envolvendo a escolha da tarefa seguida da consequência 'tela preta', e as (b) possíveis contingências envolvendo a escolha da alternativa seguida do jogo: (a) A ausência de contato social pode fazer parte da história do indivíduo, muitas vezes esses participantes podem apresentar pouco contato social com pais, parentes, etc. Uma possibilidade é que essa experiência anterior poderia explicar a escolha dessa alternativa. Lee, Belfiore, e Toro-Zambrana (2001) sugerem que fatores específicos da tarefa, como a história de trabalhar com um delineamento particular, uma forma particular de tarefa e a saliência entre as opções diferentes de tarefas, podem afetar a seleção de comportamentos dos participantes. Isto é, os indivíduos podem selecionar a opção de delineamento ou de tarefa que mais proximamente lembram uma condição que eles tiveram no passado e na qual já possuem alguma experiência, uma vez que muitas vezes não discriminam qual condição resulta em maiores níveis de reforço. Uma possibilidade adicional, é que as novas condições, representadas pela alternativa seguida do jogo, tanto pelo fator novidade, quanto por demandarem desempenho diferente do habitual, possam conter características levemente aversivas para participantes que tenham baixa tolerância a mudanças ambientais. Assim, a escolha seria controlada por reforçamento negativo ou por 'exclusão'; (b) As primeiras escolhas mais acentuadas podem ter ocorrido pelo fator 'novidade da tarefa'. Os participantes poderiam não estar discriminando entre as alternativas ainda, portanto, para avaliar os efeitos do tipo de reforçador e de sua magnitude nas condições estudadas, seria necessário, por exemplo, acentuar essas características das alternativas. Adicionalmente, poder-se-ia tentar identificar e/ou manipular as variáveis motivacionais dos participantes [(e.g., saciação e privação (Michael, 1993). Indivíduos poderiam ser privados/saciados de determinados estímulos (e.g., vídeos reforçadores) por períodos específicos para se avaliar os efeitos da manipulação das variáveis motivacionais, ao se utilizar vídeos reforçadores, na aquisição da discriminação simples e da reversão)]; (c) Para favorecer a discriminabilidade entre as alternativas, procedimentos envolvendo atraso no reforço ou durações diferentes de reforço poderiam ser incluídos (Hanna \& Blackman, 2005), ou até mesmo poderia ser realizado um treino discriminativo antes do procedimento de escolha (Escobal \& Goyos, 2015; Kodak, Lerman, Volkert, \& Trosclair, 2007); (d) No presente estudo não foi medida a latência média do desempenho nas alternativas durante a avaliação de preferência por tarefas, futuros estudos poderiam avaliar tal medida, pois a latência média de desempenho pode influenciar na escolha. $\mathrm{O}$ mesmo pode ocorrer com o tempo de duração da consequência e da 'tela preta'. Por exemplo, para determinados participantes que consideram o contato social com o experimentador reforçador, desempenhos realizados em maior tempo de latência/duração da consequência podem ser escolhidos. Para o 
participante que quer se livrar de um estímulo aversivo, a tarefa, por exemplo, poderia ser considerada aversiva ou a até mesmo própria presença do experimentador. Então, futuros estudos poderiam avaliar a consequência social ser mais reforçadora por apresentar menos contato, caso este não seja reforçador.

\section{Conclusões}

Destacando alguns pontos positivos do estudo: a ideia de que reforçadores são mais eficazes quando o indivíduo está privado deles, foi novamente realçada nesse estudo. Se o participante obteve muitos reforçadores em um passado recente, é possível que ele se sacie, e se isso ocorre, tal reforçador não será muito efetivo para motivar esse indivíduo. A utilização de uma ferramenta informatizada para a realização da avaliação do valor reforçador dos itens de preferência (MestreLibras) trouxe uma maneira diferente de realizar avaliação de preferência, com um refinamento da metodologia, além de ter inserido a realização diária de MSWOs antes de cada sessão de avaliação do valor reforçador, para aumentar a probabilidade de seleção do item reforçador naquele momento.

Em relação ao comportamento social e aos procedimentos deste estudo, Skinner, 1953/1965, define "comportamento social como comportamento de duas ou mais pessoas, uma em relação à outra, ou em conjunto em relação a um ambiente comum". Nesta pesquisa, as crianças jogavam com o experimentador no Tablet e não sozinhas. Nos jogos apresentados nesta pesquisa, uma tentativa era realizada pelo participante e uma pelo experimentador. O desempenho de um indivíduo dependia do desempenho do outro para que houvesse continuidade no jogo. Assim, essa pesquisa estimulou nas crianças comportamentos de escolher, esperar pela vez, compartilhar um item e interagir socialmente, todos fundamentais ao repertório de qualquer indivíduo. A metodologia empregada promoveu a inter-relação entre diversas áreas do conhecimento (Psicologia, Educação Especial e Informática) e viabilizou um conjunto de intervenções capaz de possibilitar uma nova forma de planejamento de ensino utilizando aplicativos para Tablet com crianças.

\section{Referências}

Andery, M. A. P. A., \& Sério, T. M. A. P. (2006). Comportamento Social. In J. H. Guilhardi \& N. C. E. Aguirre (Eds.), Sobre comportamento e cognição: Expondo a variabilidade. (pp. 124132). Santo André, SP: ESETec.

Baer, D. M., Wolf, M. M., \& Risley, T. R. (1968). Some current dimensions of applied behavior analysis. Journal of Applied Behavior Analysis, 1, 91-97. doi:10.1901/jaba.1968.1-91

Barnes, D. (1994). Stimulus equivalence and relational frame theory. The Psychological Record, 44, 91-124.

Barnes-Holmes, D., \& Barnes-Holmes, Y. (2000). Explaining complex behavior: Two perspectives on the concept of generalized operant classes. The Psychological Record, 50, 251-265.

Carr, J. E., Nicolson, A. C., \& Higbee, T. S. (2000). Evaluation of a brief multiple-stimulus preference assessment in a naturalistic context. Journal of Applied Behavior Analysis, 33, 353-357. doi:10.1901/jaba.2000.33-353

Conyers, C., Doole, A., Vause, T., Harapiak, S., Yu, D. C. T., \& Martin, G. L. (2002). Predicting the relative efficacy of three presentation methods for assessing preferences of persons with developmental disabilities. Journal of Applied Behavior Analysis, 35, 49-58. doi:10.1901/ jaba.2002.35-49

Cooper, M. J., Griffith, K. G., \& Filer, J. (1999). School intervention for inclusion of students with and without disabilities. Focus on Autism and Other Developmental Disabilities, 14, 110115. doi:10.1177/108835769901400207

Critchfield, T. S. (2011). Translational contributions of the experimental analysis of behavior. The Behavior Analyst, 34, 3-17.

De-Leon, I. G., \& Iwata, B. A. (1996). Evaluation of a multiple-stimulus presentation format for assessing reinforcer preferences. Journal of Applied Behavior Analysis, 29, 519-533. doi:10.1901/ jaba.1996.29-519

Elias, N. C., \& Goyos, C. (2010). MestreLibras no ensino de sinais: Tarefas informatizadas de escolha de acordo com o modelo e Equivalência 
de Estímulos. In E. G. Mendes \& M. A. Almeida (Eds.), Das margens ao centro: Perspectivas para as políticas e práticas educacionais no contexto da educação especial e inclusiva (pp. 223-234). São Carlos, SP: Junqueira \& Marin.

Escobal, G., \& Goyos, C. (2015). Trabalho de indivíduos com atraso no desenvolvimento intelectual: Contribuições da Análise do Comportamento Aplicada (ABA) e Processos de Tomada de Decisão. São Carlos, SP: Editora da Universidade Federal de São Carlos.

Escobal, G., Macedo, M., Duque, A. L., Gamba, J., \& Goyos, C. (2010). Contribuições do paradigma de escolha para identificação de preferências por consequências reforçadoras. In M. M. C. Hübner, M. R. Garcia, P. R. Abreu, E. N. P. De Cillo, \& P. B. Faleiros (Eds.), Sobre comportamento e cognição (pp. 371-376). Santo André, SP: ESEtec.

Forness, S., \& Kavale, K. (1999). Teaching social skills in children with learning disabilities: A meta-analysis of the research. Learning Disability Quarterly, 19, 2-13. doi:10.2307/1511048

Groskreutz, M. P., \& Graff, R. B. (2009). Evaluating pictorial preference assessment: The effect of differential outcomes on preference assessment results. Research in Autism Spectrum Disorders, 3, 113-128. doi:http://dx.doi.org/10.1016/j. rasd.2008.04.007

Hall, R. V. (1974). Managing behavior - behavior modification: The measurement of behavior. Lawrence, KS: H \& H Enterprises.

Hanna, E. S., \& Blackman, D. E. (2005). Escolha entre atrasos e durações diferentes de Reforço: Sobre a generalização de preferências em tentativas discretas para um procedimento de operante livre. Revista Brasileira de Análise do Comportamento, 1, 81-95. doi:http://dx.doi. org/10.18542/rebac.v1i1.764

Higbee, T. S., Carr, J. E., \& Harrison, C. D. (1999). The effects of pictorial versus tangible stimuli in stimulus-preference assessments. Research in Developmental Disabilities, 20(1), 63-72.

Kazdin, A. E., \& Klock, J. (1973). The effect of nonverbal teacher approval on student attentive behavior. Journal of Applied Behavior Analysis, 6(4), 643-654. doi:10.1901/jaba.1973.6-643

Keller, F. S., \& Schoenfeld, W. N. (1950). Principles of Psychology. New York: Appleton-Century-Crofts.
Kodak, T., Lerman, D. C., Volkert, V. M., \& Trosclair, N. (2007). Further examination of factors that influence preference for positive versus negative reinforcement. Journal of Applied Behavior Analysis, 40, 25-44. doi:10.1901/ jaba.2007.151-05

Lee, D. L., Belfiore, P. J., \& Toro-Zambrana, W. (2001). Programming for efficiency: The effects of motion economy on vocational tasks for adults with severe and profound mental retardation. Research in Developmental Disabilities, 16, 205-220. doi:10.1016/0891-4222(95)00009-C

Lerman, D. C., Iwata, B. A., Rainville, B., Adelinis, J. D., Crosland, K., \& Kogan, J. (1997). Effects of reinforcement choice on task responding in individuals with developmental disabilities. Journal of Applied Behavior Analysis, 30, 411422. doi:10.1901/jaba.1997.30-411

Mace, F. C., \& Critchfield, T. S. (2010). Translational research in behavior analysis: Historical traditions and imperative for the future. Journal of the Experimental Analysis of Behavior, 93, 293312. doi:10.1901/jeab.2010.93-293

Michael, J. (1993). Establishing operations. The Behavior Analyst, 16, 191-206.

Nuernberger, J. E., Smith, C. A., Czpar, K. N., \& Klatt, K. P. (2012). Assessing preference for social interaction in children diagnosed with autism. Behavioral Interventions, 27, 33-44. doi:10.1002/bin.1336

Piazza, C. C., Fisher, W. W., Bowman, L. G., \& Blakeley-Smith, A. (1999). Identifying and assessing reinforcers using choice paradigms. In P. M. Ghezzi, W. Williams, \& J. E. Carr (Eds.), Autism: Behavior analytic perspectives (pp. 101-107). Reno, NV: Context Press.

Poling, A. (2010). Looking to the future: Will behavior analysis survive and prosper? The Behavior Analyst, 33, 6-17.

Sampaio, A. A. S., \& Andery, M. A. P. A. (2010). Comportamento social, produção agregada e prática cultural: Uma análise comportamental de fenômenos sociais. Psicologia: Teoria e Pesquisa, 26(1), 83-192. Recuperado em http://www. scielo.br/pdf/ptp/v26n1/a20v26n1

Skinner, B. F. (1965). Science and human behavior. New York: Free Press. (Original publicado em 1953) 
Skinner, B. F. (1992). Verbal behavior. Acton, MA: Copley. (Original publicado em 1957)

Smith, J. D. (2012). Single-Case Experimental Designs: A systematic review of published research and current standards. Psychological Methods, 17, 510-550. doi:10.1037/a0029312

Snyder K., Higbee, T. S., \& Dayton, E. (2012). Preliminary investigation of a video-based stimulus preference assessment. Journal of Applied Behavior Analysis, 45(2), 413-418. doi:10.1901/ jaba.2012.45-413

Spence, S. H., Donovan, C., \& Brechman-Toussaint, M. (1999). Social skills, social outcomes, and cognitive features of childhood social phobia. Journal of Abnormal Child Psychology, 108, 211-221. doi:10.1037/0021-843X.108.2.211

Reid, D. H., Parsons, M. B., Green, C. W., \& Browning, L. B. (2001). Increasing one aspect of self-determination among adults with severe multiple disabilities in supported work. Journal of Applied Behavior Analysis, 34, 341-344. doi:10.1901/jaba.2001.34-341
Tawney, J. W., \& Gast, D. L. (1984). Single subject research in special education. Columbus, $\mathrm{OH}$ : Charles E. Merril.

Van Houten, R., Nau, P. A., Mackenzie-Keating, S. E., Sameoto, D., \& Colavecchia, B. (1982). An analysis of some variables influencing the effectiveness of reprimands. Journal of Applied Behavior Analysis, 15(1), 65-83. doi:10.1901/ jaba.1982.15-65

Vollmer, T. R., \& Hackenberg, T. D. (2001). Reinforcement contingencies and social reinforcement: Some reciprocal relations between basic and applied research. Journal of Applied Behavior Analysis, 34, 241-253. doi:10.1901/ jaba.2001.34-241

Recebido: 29/03/2016

$1^{a}$ revisão: 23/08/2016

$2^{a}$ revisão: 31/08/2016

$3^{a}$ revisão: $1 \% 09 / 2016$

Aceite final: 02/09/2016 